\title{
DOSES DE LODO DE ESGOTO NA FERTILIDADE DO SOLO APÓS A COLHEITA DO GIRASSOL
}

\section{DOSES OF SEWAGE SLUDGE ON SOIL FERTILITY AFTER SUNFLOWER HARVESTING}

\author{
Thomaz Figueiredo LOBO ${ }^{1}$ \\ Ricardo Alexandre LAMBERT ${ }^{2}$ \\ Helio GRASSI FILHO ${ }^{3}$
}

\begin{abstract}
RESUMO
O uso agrícola do lodo de esgoto como adubo orgânico é considerada hoje como alternativa mais promissora de disposição final deste resíduo, devido a sua sustentabilidade e seu efeito pode ser potencializado, aliando-se utilização agrícola e recuperação de áreas degradadas. O objetivo deste trabalho foi avaliar o efeito do lodo de esgoto na fertilidade do solo em diferentes profundidades no perfil do solo após o primeiro ano de aplicação deste resíduo. O experimento foi conduzido na Fazenda Experimental de São Manuel da Faculdade de Ciências Agronômicas da UNESP Campus de Botucatu. Adotou-se o delineamento experimental em blocos casualizados constituído por 6 tratamentos e 5 repetições. Os tratamentos foram: T0 - sem N; T1 - $50 \mathrm{~kg}$ de N ha-1 da adubação mineral; T2 - $25 \mathrm{~kg}^{-1 e ~ N}$ ha $^{-1}$ do lodo de esgoto e $25 \mathrm{~kg}$ ha ${ }^{-1}$ de adubação mineral; T3 - $50 \mathrm{~kg}$ de $\mathrm{N} \mathrm{ha}{ }^{-1}$ do lodo de esgoto; T4 - $75 \mathrm{~kg}$ de N ha- ${ }^{-1}$ do lodo de esgoto ; T5 - $100 \mathrm{~kg}$ de N ha ${ }^{-1}$ do lodo de esgoto. A avaliação da fertilidade do solo foi efetuada após 4 meses das aplicações do lodo de esgoto. Não houve alterações no $\mathrm{pH}, \mathrm{MO}, \mathrm{CTC}, \mathrm{V} \%, \mathrm{Mg}, \mathrm{SB}$ e $\mathrm{Mn}$, em todas as profundidades do solo em função da aplicação de lodo de esgoto. A aplicação do lodo de esgoto incrementou os teores de $\mathrm{H}+\mathrm{Al}, \mathrm{S}, \mathrm{Cu}, \mathrm{Fe}$ e $\mathrm{Zn}$, do solo na camada superficial $(0-0,10$ $\mathrm{m}$ de profundidade), entretanto não afetou a fertilidade do solo na camada subsuperficial $(0,20-0,40 \mathrm{~m}$ de profundidade).
\end{abstract}

Palavras chaves: Biosólido; adubação e reciclagem de nutrientes.

\section{ABSTRACT}

The agricultural use of sewage sludge as organic fertilizer is currently considered the most promising alternative for disposal of such waste due to its sustainability and its effect can be enhanced by combing the agricultural use and recovery of degraded areas. The objective of this study was to evaluate the effect of sewage sludge on soil fertility at different depths in the soil profile after the first year of application of this waste. The experiment was conducted at the Experimental Farm in San Manuel Faculty of Agronomic Sciences, UNESP, Botucatu Campus. We adopted the experimental design of randomized blocks consisting of six treatments and five repetitions. The treatments were: T0 - without N; T1 $-50 \mathrm{~kg} \mathrm{~N}^{-1}$ mineral $^{-1}$ fertilization; T2 - $25 \mathrm{~kg} \mathrm{~N}^{-1}$ of sewage sludge and $25 \mathrm{~kg}^{-1}$ ha of mineral fertilization; T3 $-50 \mathrm{~kg} \mathrm{~N}^{-1} a^{-1}$ sewage sludge; T4 $75 \mathrm{~kg} \mathrm{~N} \mathrm{ha}^{-1}$ sewage sludge; T5 - $100 \mathrm{~kg} \mathrm{~N} \mathrm{ha}^{-1}$ sewage sludge. The evaluation of soil fertility was performed 4 months after the applications of sewage sludge. There was no change in $\mathrm{pH}, \mathrm{OM}, \mathrm{CEC}$ and $\mathrm{V} \%, \mathrm{Mg}, \mathrm{Mn}$ and $\mathrm{SB}$, at all depths of the soil according to the application of sewage sludge. The application of sewage sludge increased the concentration of $\mathrm{H}+\mathrm{Al}, \mathrm{S}, \mathrm{Cu}$, Fe and $\mathrm{Zn}$ in soil surface layer ( 0 to $0,10 \mathrm{~m}$ deep), however did not affect the soil fertility in the top subsurface $(0,20$ to $0,40 \mathrm{~m}$ deep).

Key-words: biosolid; fertilization and nutrient recycling.

\footnotetext{
${ }^{1}$ Doutor em Agronomia/Agricultura - Faculdade de Ciências Agronômicas de Botucatu - UNESP - Departamento de Recursos Naturais/Ciência do Solo. Rua José Barbosa de Barros $n^{\circ} 1780$, CEP 18610-307, Botucatu, SP, Brasil. E-mail: thomaz.lobo@superig.com.br. Autor para correspondência.

${ }^{2}$ Doutorando em Agronomia/Irrigação e Drenagem - Faculdade de Ciências Agronômicas de Botucatu - UNESP - Departamento de Recursos Naturais/Ciência do Solo. Rua José Barbosa de Barros n¹780, CEP 18610-307, Botucatu, SP, Brasil. E-mail: lambert@fca.unesp.br

${ }^{3}$ Professor Doutor em Agronomia - Faculdade de Ciências Agronômicas de Botucatu - UNESP - Departamento de Recursos Naturais/Ciência do Solo. Rua José Barbosa de Barros n¹780, CEP 18610-307, Botucatu, SP, Brasil. E-mail: heliograssi@fca.unesp.br
} 
LOBO, T. F. et al. Doses de lodo de esgoto...

\section{INTRODUÇÃO}

A deposição final do lodo de esgoto vem se caracterizando como um dos problemas ambientais urbanos mais relevantes da atualidade, e que cresce diariamente tanto nos países desenvolvidos quanto naqueles em desenvolvimento, com reflexos da ampliação das redes de coleta e incremento dos níveis de tratamento (Pegorini et al., 2003). Uma forma de se aproveitar esse resíduo seria sua utilização na agricultura como fonte de nutrientes.

Entretanto a maior preocupação para a utilização do lodo de esgoto na agricultura é o aumento da concentração de elementos tóxicos no solo. Vários destes elementos são essenciais para as plantas. Em concentrações tóxicas, metais e outros agentes potencialmente poluentes, podem inibir as atividades enzimáticas essenciais, induzir a geração de espécies ativas de oxigênio nas células microbianas e alterar a estrutura das comunidades microbianas. $\mathrm{Em}$ pequenas quantidades alguns metais pesados como $\mathrm{Cu}, \mathrm{Fe}, \mathrm{Mn}$ e $\mathrm{Zn}$ são indispensáveis para o desenvolvimento vegetal, no entanto quantidades elevadas podem ser tóxicas (Berton, 2000)

O lodo de esgoto é proveniente de Estações de Tratamento Biológico das águas servidas, com predominância de esgotos domésticos sobre os industriais e os níveis de metais pesados e patógenos, permanecem dentro das faixas aceitáveis para uso agronômico (Cetesb, 1999).

Melo \& Marques (1992) constataram que a CTC do solo sofreu uma elevação até 230 dias após a aplicação de lodo de esgoto, decrescendo em seguida. Em dose de $32 \mathrm{t} \mathrm{ha}^{-1}$ de lodo, Melo et al. (1994) verificaram que houve correlação entre a CTC do solo e o teor da fração húmina da $\mathrm{MO}$, por um período de 230 dias. A partir deste período, a correlação não foi significativa, talvez porque a fração húmina sofreu polimerização e não tenha contribuído com cargas para a CTC. Em última análise esse fato se deve à natureza da matéria orgânica contida no resíduo, o que condicionará a sua velocidade de decomposição no solo.

O lodo de esgoto pode ser utilizado para recuperar áreas degradadas cujos solos sofreram profundas alterações físicas e/ou químicas e consequentemente, apresentam condições impróprias ao desenvolvimento da vegetação. Normalmente aplicam-se uma única vez, quantidades relativamente elevadas de lodo de esgoto nessas áreas, chegando a atingir doses de até $495 \mathrm{t} \mathrm{ha}^{-1}$ (Shirotta \& Rocha, 1997)

Um papel importante do uso agrícola do lodo de esgoto está associado ao seu papel de condicionador de solo, função principalmente do conteúdo orgânico do resíduo. Embora a matéria orgânica em solos minerais represente menos de $5 \%$ de componentes sólidos (Silva et al., 2000), ela é responsável por cerca de $70 \%$ a $80 \%$ da capacidade de troca de cátions (CTC) em solos tropicais (Raij, 1969).

No Brasil, embora as pesquisas ainda se encontrem em fases incipientes diversos trabalhos já demonstram os benefícios da aplicação do lodo de esgoto como fornecedor de nutrientes a diversas culturas (Berton et al., 1989; Oliveira et al., 1995; Biscaia \& Miranda, 1996) e na melhoria das propriedades físicas do solo (Jorge et al., 1991).

O objetivo deste trabalho foi avaliar o efeito do lodo de esgoto na fertilidade do solo em diferentes profundidades no perfil do solo após o primeiro ano de aplicação deste resíduo.

\section{MATERIAL E MÉTODOS}

O experimento foi conduzido na Fazenda Experimental São Manuel pertencente à Faculdade de Ciências Agronômicas da UNESP de Botucatu, localizada no município de São Manuel a $\left(22^{\circ} 25^{\prime} \mathrm{S}\right.$; Latitude Sul, $\left.48^{\circ} 34^{\prime} \mathrm{W}\right)$ Longitude Oeste de Greenwich, com altitude de $750 \mathrm{~m}$. O clima da região, segundo a classificação de Köppen, é do tipo mesotérmico, Cwa, ou seja, subtropical úmido com estiagem no período de inverno e com chuvas de novembro a abril sendo a precipitação média anual do município de $1.433 \mathrm{~mm}$, com umidade relativa do ar média de $71 \%$ e temperatura média de $23^{\circ} \mathrm{C}$. A classificação e os dados meteorológicos foram fornecidos pelo Departamento de Recursos Naturais, área de Ciências Ambientais / FCA UNESP - Botucatu. No período de janeiro a abril do ano de 2005, época que foi desenvolvido a cultura, foram registrados $507 \mathrm{~mm}$ de precipitação no ciclo da cultura.

O solo da área experimental é classificado como Latossolo Vermelho Escuro (EMBRAPA,

TABELA 1 - Características químicas do solo da área experimental.

\begin{tabular}{|c|c|c|c|c|c|c|c|c|c|c|c|}
\hline \multirow{2}{*}{$\begin{array}{c}\text { Prof. } \\
\mathrm{cm}\end{array}$} & \multirow{2}{*}{$\begin{array}{c}\mathrm{pH} \\
\mathrm{CaCl}_{2}\end{array}$} & \multirow{2}{*}{$\begin{array}{l}\text { M.O. } \\
\text { g dm }^{-3}\end{array}$} & \multirow{2}{*}{$\begin{array}{c}\mathrm{P}(\text { res. }) \\
\mathrm{mg} \mathrm{dm}^{-3}\end{array}$} & $\mathrm{H}+\mathrm{Al}$ & $\mathrm{Al}^{+3}$ & $\mathrm{~K}^{+}$ & $\mathrm{Ca}^{+2}$ & $\mathrm{Mg}^{+2}$ & SB & $T$ & V \\
\hline & & & & \multicolumn{7}{|c|}{ - } & $\%$ \\
\hline $0-20$ & 6,1 & 12 & 20 & 13 & 1 & 1,9 & 19 & 12 & 32,9 & $\begin{array}{c}45 \\
9\end{array}$ & 71 \\
\hline \multirow[t]{3}{*}{$20-40$} & 6,1 & 7 & 6 & 13 & 1 & 1,6 & 17 & 9 & 27,6 & $\begin{array}{c}40 \\
6\end{array}$ & 68 \\
\hline & & B & & & & $\mathrm{Fe}$ & & $\mathrm{Mn}$ & & $\mathrm{Zr}$ & \\
\hline & \multicolumn{11}{|c|}{------------------- $\mathrm{mg} \mathrm{dm}^{-3}$----------------- } \\
\hline $0-20$ & & 0,11 & \multicolumn{2}{|c|}{1,0} & \multicolumn{2}{|c|}{20} & & \multicolumn{2}{|l|}{7,7} & \multicolumn{2}{|c|}{1,2} \\
\hline $20-40$ & & 0,09 & \multicolumn{2}{|c|}{0,9} & \multicolumn{2}{|r|}{13} & & \multicolumn{2}{|l|}{4,1} & \multicolumn{2}{|c|}{1,2} \\
\hline
\end{tabular}


Foi adotado o delineamento experimental em blocos casualizados constituído por 6 tratamentos e 5 repetições. Os tratamentos foram: T0 - sem adubação nitrogenada (testemunha); T1 - adubação química nitrogenada de acordo com (Raij et al., 1996) (Adubação 100\% N químico); T2 - 50\% da adubação nitrogenada proveniente do lodo de esgoto e $50 \%$ na forma da adubação química, em cobertura; T3 - 100\% da adubação nitrogenada do recomendado para a cultura do girassol, proveniente do lodo de esgoto; T4 - 150\% da adubação nitrogenada proveniente do lodo de esgoto; T5 - 200\% da adubação nitrogenada proveniente do lodo de esgoto.

O cálculo do $\mathrm{N}$ proveniente do lodo de esgoto foi realizado levado em consideração a sua taxa de mineralização do nitrogênio de $30 \%$ durante o ciclo da cultura segundo norma P 4.230 da CETESB (1999).

Utilizou-se o lodo de esgoto proveniente da Estação de Tratamento de Esgoto da cidade de Jundiaí/SP. A amostragem do lodo de esgoto foi realizada dividindo-se o monte em quatro partes de onde foram retiradas sub amostras em cada parte, misturadas para formar uma única amostra, que apresentou os seguintes resultados: $\mathrm{N}-31,8 \mathrm{~g} \mathrm{~kg}^{-1}$, $\mathrm{P}_{2} \mathrm{O}_{5}-17,6 \mathrm{~g} \mathrm{~kg}^{-1}, \mathrm{~K}_{2} \mathrm{O}-1,8 \mathrm{~g} \mathrm{~kg}^{-1}$, Umidade $675,8 \mathrm{~g} \mathrm{~kg}^{-1}$, M.O. $-55 \mathrm{~g} \mathrm{~kg}^{-1}, \mathrm{C}-306 \mathrm{~g} \mathrm{~kg}^{-1}, \mathrm{Ca}-$ $12,5 \mathrm{~g} \mathrm{~kg}^{-1}, \mathrm{Mg}-2,2 \mathrm{~g} \mathrm{~kg}^{-1}, \mathrm{~S}-45,6 \mathrm{~g} \mathrm{~kg}^{-1}, \mathrm{Na}-$ $1.520 \mathrm{mg} \mathrm{kg}^{-1}, \mathrm{Cu}-812 \mathrm{mg} \mathrm{kg}^{-1}, \mathrm{Fe}-31.650 \mathrm{mg}$ $\mathrm{kg}^{-1}, \mathrm{Mn}-3.400 \mathrm{mg} \mathrm{kg}^{-1}, \mathrm{Zn}-2.150 \mathrm{mg} \mathrm{kg}^{-1}, \mathrm{C} / \mathrm{N}-$ $10, \mathrm{pH}-4,3$ determinado conforme método descrito em Brasil, (1988). Os metais pesados foram analisados no laboratório BIOAGRI e o lodo de esgoto apresentou as seguintes características: $\mathrm{Cd}-11$ $\mathrm{mg} \mathrm{kg}^{-1}, \mathrm{Cr}-111 \mathrm{mg} \mathrm{kg}^{-1}, \mathrm{Ni}-26 \mathrm{mg} \mathrm{kg}^{-1}, \mathrm{~Pb}-85$ $\mathrm{mg} \mathrm{kg}^{-1}$, conforme o método descrito por RAIJ et al. (2001).

Cada parcela foi constituída por uma área de 100,8 metros quadrados $(14 \mathrm{~m} \times 7,2 \mathrm{~m})$ com uma distância de $3 \mathrm{~m}$ de uma parcela a outra do mesmo bloco. A distância entre um bloco ao outro foi de 1,8 $\mathrm{m}$.

A cultivar utilizada foi a HELIO 251, por ser um material altamente produtivo e resistente as principais doenças.

Antes da instalação do experimento foi realizada uma gradagem com a grade aradora em área total para limpeza e incorporação das plantas daninhas na área, a profundidade de operação foi de aproximadamente $0,20 \mathrm{~m}$; aplicando-se em seguida o lodo de esgoto.

O lodo de esgoto foi aplicado em base seca nos tratamentos, utilizando-se um distribuidor de esterco, regulado na menor dosagem a ser aplicada, ou seja T2 $\left(2.470 \mathrm{~kg} \mathrm{ha}^{-1}\right)$, para o tratamento T3, adotou-se 2 passadas $\left(4.940 \mathrm{~kg} \mathrm{ha}^{-1}\right)$, para o $\mathrm{T} 4,3$ passadas $\left(7.410 \mathrm{~kg} \mathrm{ha}^{-1}\right)$ e finalmente T5 com 4 passadas $\left(9.880 \mathrm{~kg} \mathrm{ha}^{-1}\right)$.

Foi aplicado o trifuralin (ia) na dose de 1,2 $\mathrm{L} \mathrm{ha}^{-1}$ juntamente com o ácido bórico na dose $6 \mathrm{~kg}$ $\mathrm{ha}^{-1}$, buscando o controle da sementeira; adubação e semeadura.
As sementes do girassol foram semeadas em espaçamento de 0,9 m entre linhas e 4 sementes por metro linear na profundidade de $0,03 \mathrm{~m}$. A adubação de semeadura para todas as parcelas foi constituída de $30 \mathrm{~kg} \mathrm{ha}^{-1}$ de $\mathrm{P}_{2} \mathrm{O}_{5}$ na forma de superfosfato simples $\left(18 \% \mathrm{P}_{2} \mathrm{O}_{5}\right)$ e $30 \mathrm{~kg} \mathrm{ha}^{-1}$ de $\mathrm{K}_{2} \mathrm{O}$ na forma de cloreto de potássio $\left(60 \% \mathrm{~K}_{2} \mathrm{O}\right)$.

$\mathrm{A}$ adubação nitrogenada foi realizada logo após a semeadura no T1, na dose de $10 \mathrm{~kg}$ de $\mathrm{N}$ ha ${ }^{-1}$ na forma de uréia ( $45 \%$ de $\mathrm{N}$ ), que é o recomendado para o Estado de São Paulo. Os tratamentos T0, T2, T3, T4 e T5 não receberam esta adubação com $\mathrm{N}$ na semeadura.

Os tratamentos que receberam a adubação de cobertura foram os tratamentos T1 e T2. O tratamento T1 recebeu $40 \mathrm{~kg}$ de $\mathrm{N} \mathrm{ha}^{-1}$ que é o recomendado para o Estado de São Paulo, de acordo com (Raij et al., 1996), o tratamento T2 recebeu 25 $\mathrm{kg}$ de $\mathrm{N} \mathrm{ha}^{-1}$, porque os outros $25 \mathrm{~kg} \mathrm{ha}^{-1}$ foram aplicados antes do plantio na forma de lodo de esgoto, totalizando os $50 \mathrm{~kg} \mathrm{ha}^{-1}$ a mesma quantidade de $\mathrm{N}$ utilizada no $\mathrm{T} 1$, porem de maneira diferente. $\mathrm{A}$ forma utilizada para a cobertura foi à uréia. Esta operação foi efetuada aos 30 dias após a emergência e como choveu $20 \mathrm{~mm}$ após esta adubação não houve necessidade de incorporar.

A adubação foliar foi realizada com uma aplicação de $\mathrm{B}$ aos 45 dias após o plantio, na dose de $1 \mathrm{~g} \mathrm{~L}^{-1}$ de ácido bórico. Fez-se esta aplicação foliar, devido à constatação de rachaduras nas hastes do girassol, sintoma típico da falta de boro, solucionando-se o problema.

Após a colheita do girassol foram retiradas 10 sub-amostras de solo ao acaso, para formar uma amostra composta em cada parcela. As amostras foram coletadas nas entrelinhas da cultura do girassol, nas profundidades de $0-0,10 ; 0,10-0,20$ e $0,20-0,40 \mathrm{~m}$. As análises para avaliação da fertilidade foram executadas conforme o método descrito por RAIJ et al. (2001).

Os dados foram submetidos a comparação das médias, sendo utilizado o teste de Tukey a $5 \%$ de probabilidade, de acordo com os procedimentos do Statistical Analysis System (Sas Institute, 2001).

\section{RESULTADOS E DISCUSSÃO}

Observa-se nas Tabelas 2, 3 e 4 que o pH em $\mathrm{CaCl}_{2}$ não alterou em nenhuma das profundidades analisadas, tendo em vista que o pH inicial do solo já estava adequado para a cultura, não havendo necessidade de corrigir o $\mathrm{pH}$ do solo. $\mathrm{O} \mathrm{pH}$ do solo se encontrava entre 5 a 6,5 , sendo que está é a faixa ideal para um maior aproveitamento dos nutrientes para a cultura, segundo Malavolta (1987). Observa-se que nas maiores profundidades o $\mathrm{pH}$ do solo encontrava-se menor do que na superfície, embora não tenha sido feito uma estatística entre as profundidades, mas pode ser reparado que a média dos tratamentos na profundidade 0 a $0,10 \mathrm{~m}$ foi de 6,4 , aos 0,10 a $0,20 \mathrm{~m} \mathrm{5,9}$ e aos 0,20 a 0,40 m 5,3.

Gomes et al (2005) e Lopes et al., (2007) concluíram em seus trabalhos que o aumento das 
LOBO, T. F. et al. Doses de lodo de esgoto...

doses de lodo de esgoto promoveu uma diminuição

$\mathrm{NO}_{2}$ e posteriormente para $\mathrm{NO}_{3}$ através das bactédo $\mathrm{pH}$ do solo, devido à passagem do $\mathrm{NH}_{4}$ para rias nitrossomonas e nitrobacter, respectivamente.

TABELA 2 - Análise do solo da área experimental de 0 a 0,10 m de profundidade após a primeira aplicação do lodo de esgoto.

\begin{tabular}{|c|c|c|c|c|c|c|c|c|c|c|c|}
\hline \multirow[t]{2}{*}{ Trat. } & $\overline{\mathrm{pH}}$ & $\mathrm{MO}$ & $P$ & $\mathrm{H}+\mathrm{Al}$ & $\mathrm{K}$ & $\mathrm{Ca}$ & $\overline{M g}$ & $\overline{S B}$ & CTC & \multirow{2}{*}{$\begin{array}{l}\mathrm{V} \\
\%\end{array}$} & \multirow{2}{*}{$\begin{array}{c}\mathrm{S} \\
\mathrm{mg} \mathrm{dm}^{-3}\end{array}$} \\
\hline & $\mathrm{CaCl}_{2}$ & $\mathrm{~g} \mathrm{dm}^{-3}$ & $\mathrm{mg} \mathrm{dm}^{-3}$ &.-- & - & $--m m$ & $\mathrm{dm}^{-3}$ & - & --- & & \\
\hline $\mathrm{T}_{0}$ & 6,5 & 21 & 19 & $11 \mathrm{~b}$ & $1,3 \mathrm{~b}$ & 23 & 10 & 35 & 46,2 & 75 & $3,0 \mathrm{~b}$ \\
\hline $\mathrm{T}_{1}$ & 6,3 & 15 & 18 & $13 \mathrm{ab}$ & $1,5 \mathrm{ab}$ & 21 & 10 & 33 & 46,2 & 72 & $3,4 \mathrm{~b}$ \\
\hline $\mathrm{T}_{2}$ & 6,5 & 18 & 19 & $12 a b$ & $1,6 \mathrm{ab}$ & 25 & 9 & 34 & 46,4 & 75 & $3,8 \mathrm{~b}$ \\
\hline $\mathrm{T}_{3}$ & 6,3 & 21 & 27 & $12 a b$ & 1,9 a & 24 & 9 & 36 & 48,0 & 73 & $5,2 a b$ \\
\hline $\mathrm{T}_{4}$ & 6,2 & 18 & 23 & $13 a$ & $1,8 \mathrm{a}$ & 24 & 9 & 34 & 47,0 & 73 & $5,8 a b$ \\
\hline$T_{5}$ & 6,3 & 20 & 25 & $13 a$ & $1,6 \mathrm{ab}$ & 26 & 10 & 36 & 49,6 & 74 & $11,4 a$ \\
\hline $\mathrm{F}$ & $1,54^{\text {ns }}$ & $1,59^{\text {ns }}$ & $3,22^{\text {ns }}$ & 2,91 & 4,3 & $0,44^{\text {ns }}$ & $0,64^{\text {ns }}$ & $0,1^{\text {ns }}$ & $0,19^{\text {ns }}$ & $0,26^{\text {ns }}$ & 4,8 \\
\hline M & 6,4 & 19,0 & 22,0 & 12,4 & 1,6 & 24,0 & 9,7 & 35 & 47,3 & 73,8 & 5,4 \\
\hline DMS & & & & 1,52 & 0,44 & & & & & & 5,75 \\
\hline $\mathrm{CV}$ & 3 & 22 & 22 & 7 & 13 & 23 & 18 & 21 & 15 & 6 & 582 \\
\hline
\end{tabular}

ns-não significativo; *-significativo, a 5\%; M-média; CV-coeficiente de variação, Trat.-tratamento

Lobo \& Grassi Filho (2009) constataram que os teores foliares de $\mathrm{N}$ neste mesmo experimento apresentaram adequados para todos os tratamentos, devido a isto o lodo de esgoto supriu a necessidade da cultura em $\mathrm{N}$.

Para o teor de Matéria Orgânica (M.O.) verifica-se nas Tabelas 2, 3 e 4 que não diferiram estatisticamente em nenhuma das profundidades analisadas. No primeiro ano de aplicação de lodo não se observa diferença esta diferença ira ser observada ao longo do tempo com aplicações sucessivas. Bataglia et al. (1983) detectaram aumento significativo no teor de matéria orgânica do solo, após a aplicação de lodo de esgoto em vasos com solo Argissolo Vermelho Amarelo, cultivado com capim braquiaria. Dias (2005) observou que houve uma tendência de aumento do teor de matéria orgânica no solo na profundidade de 0,20 a 0,40 m em função da dose de lodo de esgoto após $06^{\circ}$ ano de aplicação.

$\mathrm{Na}$ Tabela 2 verifica-se que o $\mathrm{P}$ na profundidade de 0 a $0,10 \mathrm{~m}$ estatisticamente não variou, porém houve uma tendência de aumento em função das doses aplicadas, podendo-se observar que os tratamentos 3,4 e 5 , nos primeiros $0,10 \mathrm{~m}$ de profundidade, já apresentam outra classe de recomendação de adubação para culturas anuais no Estado de São Paulo, segundo Raij et al., (2001). Araújo et al., (2009) observaram um aumento no teor de $\mathrm{P}$ no solo quando aplicou o lodo de esgoto.

Observa-se na Tabela 2 que a CTC não variou nas profundidades de 0 a $0,10 \mathrm{~m}$, porém houve uma tendência de aumento nas maiores doses aplicadas, no entanto não houve diferença estatística porque as doses foram pequenas, a maior dose foi de $9,9 \mathrm{t} \mathrm{ha}^{-1}$ de matéria seca, para cau- sar aumento na CTC de um solo esta quantidade ainda é pequena. Em todas as profundidades analisadas houve esta tendência de aumento, embora todos estes aumentos não tenham sido significativos. Silva et al., (2001) observaram que com o aumento da dose de lodo de esgoto a CTC do solo aumentou-se de forma linear.

$\mathrm{Na}$ Tabela 2 verifica-se que a porcentagem de saturação de bases da CTC a pH 7,0 (V\%) não foi significativamente alterada com as doses de lodo de esgoto uma vez que o solo já apresentava valores de $\mathrm{V} \%$ elevado antes do plantio. Observase que a porcentagem de saturação de bases da CTC a pH 7,0 (V\%) na profundidade de 0,10 m é superior aos valores encontrados em profundidades maiores, sendo este um comportamento normal. Trannin (2004) não observou diferenças significativas com o aumento de dose de lodo de esgoto na V\% do solo. Observa-se na Tabela 2 que os teores de Ca não diferiram em função dos tratamentos na profundidade de 0 a $0,10 \mathrm{~m}$, diferindo somente em profundidades de 0,10 a 0,20 m (Tabela 3). Comparando os teores iniciais de Ca no solo, com os teores observados após a aplicação dos tratamentos, observa-se que não houve diferença estatísticas significativa.

Pode-se observar nas Tabelas 2, 3 e 4 que os teores de $\mathrm{Mg}$ não diferiram em todas as profundidades, porém quando comparados aos teores iniciais de $\mathrm{Mg}$ no solo com os resultados obtidos, verifica-se que os teores iniciais eram superiores, fato justificado provavelmente pela grande quantidade absorvida pela planta, ou, por este ter sido lixiviado.

Nas Tabelas 2, 3 e 4 os teores de $\mathrm{K}$ em todas as profundidades estão bem inferiores aos 
teores iniciais no solo, tendo em vista a alta capacidade do girassol em absorver o $\mathrm{K}$, segundo Castro (2005), porém grande parte deste K retorna ao solo, pelos caules, folhas e capítulos. Quando da coleta de solo, as plantas ainda estavam de pé, portanto não sendo analisado a quantidade de $\mathrm{K}$ retornado ao solo, através do caule, folhas e capítulos. O lodo de esgoto apresenta quantidade muito baixa deste elemento, conforme citado por Miller (1973). Observa-se que os teores de $\mathrm{K}$ somente diferiram nas

TABELA 3 - Analise do solo da área experimental de 0,10 a 0,20 m de profundidade após a primeira aplicação do lodo de esgoto.

\begin{tabular}{|c|c|c|c|c|c|c|c|c|c|c|c|}
\hline \multirow[t]{2}{*}{ Trat. } & $\mathrm{pH}$ & $\mathrm{MO}$ & $P$ & $\mathrm{H}+\mathrm{Al}$ & $\bar{K}$ & $\mathrm{Ca}$ & $\mathrm{Mg}$ & SB & \multirow[t]{2}{*}{ CTC } & \multirow{2}{*}{$\begin{array}{l}\mathrm{V} \\
\%\end{array}$} & \multirow{2}{*}{$\begin{array}{c}\mathrm{S} \\
\mathrm{mg} \mathrm{dm}\end{array}$} \\
\hline & $\mathrm{CaCl}_{2}$ & $\mathrm{~g} \mathrm{dm}^{-3}$ & $\mathrm{mg} \mathrm{dm}^{-3}$ & & ------- & $---m m c$ & $\mathrm{~m}^{-3}-$ & ------ & & & \\
\hline$\overline{\mathrm{T}_{0}}$ & 5,9 & 12 & 14 & 12 & 1,26 & $16 \mathrm{~b}$ & 8 & 26 & 38,4 & 67 & 3,2 \\
\hline $\mathrm{T}_{1}$ & 5,8 & 15 & 9 & 13 & 1,34 & $16 \mathrm{~b}$ & 8 & 26 & 39,2 & 66 & 4,6 \\
\hline $\mathrm{T}_{2}$ & 5,9 & 14 & 11 & 13 & 1,28 & $18 a b$ & 9 & 28 & 41,6 & 68 & 4,6 \\
\hline $\mathrm{T}_{3}$ & 6,0 & 13 & 13 & 12 & 1,52 & $18 a b$ & 8 & 28 & 40,4 & 68 & 4,8 \\
\hline $\mathrm{T}_{4}$ & 5,9 & 15 & 17 & 13 & 1,48 & $23 a$ & 9 & 33 & 46,2 & 71 & 5,6 \\
\hline $\mathrm{T}_{5}$ & 5,8 & 14 & 10 & 13 & 1,24 & $19 a b$ & 8 & 29 & 42,2 & 68 & 9,0 \\
\hline $\mathrm{F}$ & $0,2^{\text {ns }}$ & $0,6^{\mathrm{ns}}$ & $1,7^{\mathrm{ns}}$ & $0,5^{\mathrm{ns}}$ & $1,2^{\mathrm{ns}}$ & 2,8 & $0,2^{\mathrm{ns}}$ & $1,9^{\text {ns }}$ & $2,7^{\text {ns }}$ & $0,5^{\mathrm{ns}}$ & $0,5^{\mathrm{ns}}$ \\
\hline M & 5,9 & 13,8 & 12,6 & 12,9 & 1,35 & 18 & 8,4 & 28 & 41,3 & 68,2 & 5,3 \\
\hline DMS & & & & & & 5,94 & & & & & \\
\hline CV & 5 & 21 & 41 & 10 & 18 & 18 & 15 & 15 & 9 & 7 & 67 \\
\hline
\end{tabular}

ns-não significativo; *-significativo, a 5\%; M-média; CV-coeficiente de variação, Trat.-tratamento

Não houve diferença significativa entre tratamentos para a soma de bases (SB) em nenhuma das profundidades como se observa nas Tabelas 2, 3 e 4, porém houve uma tendência de aumento, o que leva a crer que nas próximas aplicações estas diferenças vão ser melhor definidas.

Quanto ao $\mathrm{H}^{+}+\mathrm{Al}^{+3}$ observa-se nas Tabelas 2,3 e 4 que houve diferença significativa nas profundidades de 0 a $0,10 \mathrm{~m}$, em função do aumento das doses do lodo de esgoto, corroborando os resultados de Trannin (2004) que observou aumento do $\mathrm{H}+\mathrm{Al}$ com aumento das doses de lodo de esgoto.

Verifica-se na Tabela 2 que o pH do solo não apresentou diferença estatística entre os tratamentos e quanto ao $\mathrm{H}+\mathrm{Al}$ do solo os dois tratamentos de maior dose de lodo (T4 e T5) apresentaram superiores ao tratamento que não recebeu $\mathrm{N}$ (T0), este fato pode ser explicado que o lodo de esgoto proporcionou um aumento de cargas negativas do solo. $\mathrm{E}$ quando é determinado o $\mathrm{H}+\mathrm{Al}$ do solo grande parte esta adsorvido nas cargas negativas. E o $\mathrm{pH}$ do solo é determinado na solução do solo.

Para o $\mathrm{SO}_{4}^{-2}$ (enxofre) observa-se na Tabela 2 que de 0 a $0,10 \mathrm{~m}$ de profundidade o tratamento 5 foi superior a todos e estatisticamente igual aos tratamentos 3 e 4 , já o tratamento 4 foi igual aos demais, porém houve uma tendência de aumento com as doses de lodo de esgoto, uma vez que o lodo de esgoto é rico em $\mathrm{SO}_{4}^{-2}$, até superior ao $\mathrm{N}$. Já na profundidade de 0,10 a 0,20 m não houve diferença significativa, no entanto houve uma tendência de aumento com as doses de lodo de esgoto. Já nas profundidades de 0,20 a $0,40 \mathrm{~m}$ não houve diferenças significativas, podendo-se observar que em profundidades maiores os teores de
$\mathrm{SO}_{4}{ }^{-2}$ no solo foram superiores. Este fato pode ter ocorrido em função da aplicação do superfosfato simples no plantio uma vez que este contém $\mathrm{SO}_{4}{ }^{-2}$, sendo este elemento menos fixado que o $\mathrm{P}$, com isso o $\mathrm{P}$ é fixado na superfície e o $\mathrm{SO}_{4}^{-2}$ na subsuperfície carregando muitas vezes o $\mathrm{Ca}$.

Quanto aos coeficientes de variação elevados apresentados para $\mathrm{S}$ em todas as profundidades de solos analisados, podem ser explicados por uma grande variação desses elementos no solo, mesmo após a aplicação do lodo de esgoto, e acreditamos que $0 n^{\circ}$ de sub amostras, para este elemento deveria ter sido maiores do que as 10 , para obtermos um resultado mais homogêneo entre as amostras.

Simonete et al., (2003), utilizando doses de até $50 \mathrm{t} \mathrm{ha}^{-1}$ de lodo de esgoto proveniente de digestão anaeróbica, sem tratamento químico, em um Argissolo Vermelho- Amarelo em vasos cultivados com milho, concluíram que a aplicação do lodo de esgoto aumentou os teores de MO, P, K, Ca, Mg, S e $\mathrm{H}+\mathrm{Al}$ e diminuiu o $\mathrm{pH}$ do solo.

$\mathrm{Na}$ Tabela 5 são apresentados os teores de $B$, que foram significativos na profundidade de 0 a $0,10 \mathrm{~m}$, para o tratamento com maior dose de lodo de esgoto em comparação com o tratamento com menor dose de lodo de esgoto, verificando-se que no tratamento com a menor dose o teor de B foi inferior. O lodo de esgoto por ser um material orgânico apresenta uma boa quantidade de $B$, embora tenha sido aplicado acido bórico na cultura do girassol por ser uma cultura extremamente exigente neste elemento. Observa-se que os teores de B iniciais no solo estavam bem abaixo do que os teores após a colheita em todos os tratamentos. 
LOBO, T. F. et al. Doses de lodo de esgoto...

TABELA 4 - Análise do solo da área experimental de 0,20 a $0,40 \mathrm{~m}$ de profundidade após a primeira aplicação do lodo de esgoto.

\begin{tabular}{|c|c|c|c|c|c|c|c|c|c|c|c|}
\hline \multirow[t]{2}{*}{ Trat. } & $\overline{\mathrm{pH}}$ & $\mathrm{MO}$ & $\bar{P}$ & $\overline{\mathrm{H}+\mathrm{Al}}$ & $\bar{K}$ & $\mathrm{Ca}$ & $\mathrm{Mg}$ & $\mathrm{SB}$ & CTC & $\mathrm{V}$ & $S$ \\
\hline & $\mathrm{CaCl}_{2}$ & $\mathrm{~g} \mathrm{dm}^{-3}$ & $\underset{\mathrm{dm}^{-3}}{\mathrm{mg}}$ & \multicolumn{6}{|c|}{$\mathrm{mmol}_{\mathrm{c}} \mathrm{dm}^{-3}$} & $\%$ & $\mathrm{mg}_{-3} \mathrm{dm}$ \\
\hline $\mathrm{T}_{0}$ & 5,3 & 12 & $8 \mathrm{ab}$ & 15 & 1,26 & 11 & 7 & 19 & 34,2 & 56 & 5,4 \\
\hline $\mathrm{T}_{1}$ & 5,1 & 12 & $4 \mathrm{~b}$ & 18 & 1,34 & 12 & 8 & 21 & 38,8 & 55 & 12,2 \\
\hline $\mathrm{T}_{2}$ & 5,2 & 13 & $5 \mathrm{ab}$ & 16 & 1,34 & 14 & 8 & 22 & 38,8 & 59 & 9,6 \\
\hline $\mathrm{T}_{3}$ & 5,4 & 12 & $7 \mathrm{ab}$ & 15 & 1,56 & 14 & 8 & 24 & 39,2 & 62 & 11,0 \\
\hline $\mathrm{T}_{4}$ & 5,5 & 15 & $12 a$ & 16 & 1,68 & 17 & 8 & 26 & 41,4 & 63 & 11,4 \\
\hline $\mathrm{T}_{5}$ & 5,4 & 14 & $4 \mathrm{~b}$ & 15 & 1,56 & 15 & 9 & 25 & 40,8 & 62 & 16,4 \\
\hline $\mathrm{F}$ & $0,65^{\text {ns }}$ & $1,22^{\text {ns }}$ & $3,422^{\circ}$ & $1,164^{n}$ & $1,39^{\text {ns }}$ & $1,53^{\mathrm{ns}}$ & $1,07^{\mathrm{ns}}$ & $1,5^{\mathrm{ns}}$ & $2,24^{\mathrm{ns}}$ & $1,09^{n s}$ & $1,485^{n}$ \\
\hline M & 5,3 & 13,03 & 6,63 & 15,7 & 1,46 & 13,83 & 7,9 & 23 & 38,86 & 59,37 & 11,0 \\
\hline DMS & & & 6,71 & & & & & & & & \\
\hline $\mathrm{CV}$ & 8 & 17 & 56 & 14 & 21 & 24 & 22 & 21 & 10 & 12 & 60 \\
\hline
\end{tabular}

ns-não significativo; *-significativo, a 5\%; M-média; CV-coeficiente de variação, Trat.-tratamento

TABELA 5 - Análise química de micronutrientes do solo de 0 a $0,10 \mathrm{~m}$ de profundidade após o primeiro cultivo do girassol com aplicação de lodo de esgoto

\begin{tabular}{cccccc}
\hline Tratamentos & $\mathrm{B}$ & $\mathrm{Cu}$ & $\begin{array}{c}\mathrm{Fe} \\
\mathrm{mg} \mathrm{dm}^{-3}\end{array}$ & $\mathrm{Mn}$ & $\mathrm{Zn}$ \\
\hline $\mathrm{T}_{0}$ & $0,192 \mathrm{ab}$ & $1,42 \mathrm{c}$ & $10,4 \mathrm{~b}$ & 12,1 & $2,4 \mathrm{~b}$ \\
$\mathrm{~T}_{1}$ & $0,146 \mathrm{ab}$ & $1,48 \mathrm{c}$ & $11,6 \mathrm{~b}$ & 10,98 & $2,12 \mathrm{~b}$ \\
$\mathrm{~T}_{2}$ & $0,138 \mathrm{~b}$ & $1,76 \mathrm{bc}$ & $12,4 \mathrm{~b}$ & 11,12 & $2,96 \mathrm{~b}$ \\
$\mathrm{~T}_{3}$ & $0,180 \mathrm{ab}$ & $2,14 \mathrm{bc}$ & $16,2 \mathrm{~b}$ & 12,84 & $3,34 \mathrm{~b}$ \\
$\mathrm{~T}_{4}$ & $0,192 \mathrm{ab}$ & $2,58 \mathrm{ab}$ & $17,2 \mathrm{ab}$ & 13,68 & $4,3 \mathrm{ab}$ \\
$\mathrm{T}_{5}$ & $0,212 \mathrm{a}$ & $3,36 \mathrm{a}$ & $25,4 \mathrm{a}$ & 13,68 & $7,2 \mathrm{a}$ \\
\hline $\mathrm{F}$ & $3,323^{\circ}$ & $15,678^{*}$ & $8,38^{*}$ & $2,38^{\text {ns }}$ & $4,841^{*}$ \\
Média & 0,177 & 2,12 & 15,53 & 12,4 & 3,72 \\
DMS & 0,06 & 0,76 & 7,74 & & 3,45 \\
$\mathrm{CV}$ & 20,8 & 19,8 & 27,4 & 14,0 & 51,1 \\
\hline
\end{tabular}

Nas Tabelas 6 e 7 observa-se que não houve diferença significativa entre os tratamentos nas maiores profundidades. Observa-se que nos tratamentos 4 e 5 que receberam doses maiores de lodo de esgoto, os maiores teores de B estão nos primeiros $0,10 \mathrm{~m}$, comparado com 0,10 a $0,20 \mathrm{~m}$ e nos tratamentos que receberam doses menores de lodo de esgoto ou não receberam os teores de B se encontram menores nos primeiros $0,10 \mathrm{~m}$, levantando-se a hipótese de que o lodo de esgoto não dei- xou o B ser lixiviado. Nas profundidades de 0 a 0,10 $\mathrm{m}$ somente o tratamento que recebeu doses mais elevadas de lodo de esgoto apresentou teores médios de $\mathrm{B}$, os outros tratamentos ficaram com teores baixos, segundo Raij et al (2001), tendo em vista que o girassol exporta até $6 \mathrm{~kg} \mathrm{ha}^{-1}$ deste elemento como observou Castro (2005). Comparando os teores iniciais deste elemento observa-se que houve um incremento no solo. 
LOBO, T. F. et al. Doses de lodo de esgoto...

TABELA 6 - Análise química de micronutrientes do solo de 0,10 a $0,20 \mathrm{~m}$ de profundidade após o primeiro cultivo do girassol e aplicação de lodo de esgoto

\begin{tabular}{cccccc}
\hline Tratamentos & $\mathrm{B}$ & $\mathrm{Cu}$ & $\begin{array}{c}\mathrm{Fe} \\
\mathrm{mg} \mathrm{dm}^{-3}\end{array}$ & $\mathrm{Mn}$ & $\mathrm{Zn}$ \\
\hline $\mathrm{T}_{0}$ & 0,186 & 1,4 & $9,8 \mathrm{~b}$ & 9,42 & 1,84 \\
$\mathrm{~T}_{1}$ & 0,154 & 1,6 & $12,2 \mathrm{ab}$ & 8,54 & 1,7 \\
$\mathrm{~T}_{2}$ & 0,168 & 1,64 & $13,0 \mathrm{ab}$ & 8,68 & 2,48 \\
$\mathrm{~T}_{3}$ & 0,216 & 1,78 & $12,8 \mathrm{ab}$ & 10,0 & 1,96 \\
$\mathrm{~T}_{4}$ & 0,186 & 1,96 & $15,4 \mathrm{a}$ & 11,76 & 2,62 \\
$\mathrm{~T}_{5}$ & 0,168 & 1,74 & $12,0 \mathrm{ab}$ & 9,12 & 2,0 \\
\hline $\mathrm{F}$ & $1,063^{\text {ns }}$ & $0,823^{\text {ns }}$ & 3,29 & $2,27^{\text {ns }}$ & $1,532^{\text {ns }}$ \\
Média & 0,18 & 1,69 & 12,53 & 9,59 & 2,1 \\
DMS & & & 4,05 & & 31,5 \\
CV & 26 & 27,5 & 17,8 & 18,4 & \\
\hline
\end{tabular}

TABELA 7 - Análise química de micronutrientes do solo de 0,20 a $0,40 \mathrm{~m}$ de profundidade após o primeiro

\begin{tabular}{cccccc}
\hline Tratamentos & $\mathrm{B}$ & $\mathrm{Cu}$ & $\begin{array}{c}\mathrm{Fe} \\
\mathrm{mg} \mathrm{dm}^{-3}\end{array}$ & $\mathrm{Mn}$ & $\mathrm{Zn}$ \\
\hline $\mathrm{T}_{0}$ & 0,176 & 1,30 & 15,0 & 8,56 & 1,34 \\
$\mathrm{~T}_{1}$ & 0,146 & 1,38 & 11,8 & 5,32 & 1,20 \\
$\mathrm{~T}_{2}$ & 0,172 & 1,60 & 12,0 & 6,32 & 1,50 \\
$\mathrm{~T}_{3}$ & 0,178 & 1,28 & 11,6 & 6,60 & 1,30 \\
$\mathrm{~T}_{4}$ & 0,182 & 1,66 & 15,8 & 8,82 & 2,02 \\
$\mathrm{~T}_{5}$ & 0,148 & 1,36 & 11,2 & 4,86 & 1,22 \\
\hline $\mathrm{F}$ & $1,139^{\text {ns }}$ & $3,22^{\text {ns }}$ & $2,08^{\text {ns }}$ & $3,24^{\text {ns }}$ & $2,676^{\text {ns }}$ \\
Média & 0,167 & 1,43 & 12,9 & 6,75 & 1,43 \\
$\mathrm{CV}$ & 19,5 & 14,0 & 23,7 & 30,1 & 29,4 \\
\hline
\end{tabular}

$\mathrm{Na}$ Tabela 5, pode ser observado que o $\mathrm{Cu}$ no solo variou significativamente nos primeiros 0,10 $\mathrm{m}$ do solo com o aumento da dose de lodo de esgoto. Este aumento foi devido ao lodo de esgoto apresentar um alto teor de $\mathrm{Cu}$. Observa-se que em todos os tratamentos teores altos acima de $0,8 \mathrm{mg}$ $\mathrm{dm}^{-3}$. Inicialmente o solo já apresentava teor alto deste nutriente, segundo Raij et al., (2001). Bertin \& Bellingieri, 2003 concluíram que o aumento nas doses de lodo, propicia um incremento dos teores de Cu no solo.

$\mathrm{Na}$ Tabela 5 verifica-se que os teores de Fe no solo na maior dose de lodo de esgoto foi superior em relação às doses de $100 \% \mathrm{~N}$ do lodo, $50 \%$ do $\mathrm{N}$ lodo com $50 \% \mathrm{~N}$ mineral, $100 \% \mathrm{~N}$ mineral e sem $\mathrm{N}$ nas profundidades de 0 a $0,10 \mathrm{~m}$. $\mathrm{Na}$ Tabela 7 pode ser verificado que na profundidade de 0,20 a 0,40 m não ocorreu variação no teor de $\mathrm{Fe}$, com isso pode ser dito que o Fe não foi lixiviado nas profundidades acima de $0,20 \mathrm{~m}$. O lodo de esgoto apresenta um alto teor de $\mathrm{Fe}$ e o tratamento que recebeu a maior quantidade de lodo de esgoto foi aplicado $308 \mathrm{~kg} \mathrm{ha}^{-1}$ de $\mathrm{Fe}$, somente proveniente do lodo de esgoto.

$\mathrm{Na}$ Tabela 5 , pode ser observado que o teor de $\mathrm{Mn}$ nos primeiros $0,10 \mathrm{~m}$ de profundidade não houve diferença significativa entre os tratamentos, mas houve uma tendência de aumento com a maior dose de lodo de esgoto.

Observa-se na Tabela 5 que o $\mathrm{Zn}$ variou na profundidade de 0 a $0,10 \mathrm{~m}$ com o aumento das doses de lodo de esgoto, tendo em vista que o lodo de esgoto apresenta elevado teor deste nutriente. Inicialmente o solo já apresentava teores elevados de $\mathrm{Zn}$. Nas Tabelas 6 e 7 pode ser verificado que os teores de $\mathrm{Zn}$ não foram alterados em função do aumento das doses de lodo de esgoto, ou seja, o Zn não se movimentou no perfil do solo em profundidades maiores de 0,10 m. Silva et al., (1998) verificaram que o lodo de esgoto é fonte de $\mathrm{Zn}$. 
LOBO, T. F. et al. Doses de lodo de esgoto...

\section{CONCLUSÕES}

- Não houve alterações no pH, MO, CTC, V\%, Mg, $\mathrm{SB}$ e $\mathrm{Mn}$, em todas as profundidades do solo em função da aplicação de lodo de esgoto.
- A aplicação do lodo de esgoto incrementou os teores de $\mathrm{H}+\mathrm{Al}, \mathrm{S}, \mathrm{Cu}$, Fe e $\mathrm{Zn}$, do solo na camada superficial $(0-0,10 \mathrm{~m}$ de profundidade), entretanto não afetou a fertilidade do solo na camada subsuperficial $(0,20-0,40 \mathrm{~m}$ de profundidade $)$

\section{REFERÊNCIAS}

1. ARAUJO, F. F. de; GIL, F. F.; TIRITAN, C. S. Lodo de esgoto na fertilidade do solo, na nutrição de Brachiaria decumbens e na atividade da desidrogenase. Pesquisa Agropecuária Tropical, Goiania, v. 39, n.1. p. 1-6, 2009.

2. BATAGLIA, O.C. et al. Resíduos orgânicos como fonte de nitrogênio para capim braquiaria. Revista Brasileira de Ciência do Solo, Viçosa, v.7, n.3, p.277-284, 1983.

3. BERTIN, E.G.; BELLINGIERI, P.A. Efeito da aplicação de lodo de esgoto, escoria de aciaria e calcário nas propriedades químicas de um solo cultivado com milho (Zea mays L.). Cientifica, Jaboticabal, v. 31, n.1. p. 81-90, 2003.

4. BERTON, R.S. Risco de contaminação do agroecossitema com metais pesados. In: BETIOL, W \& CAMARGO, O. A (eds.) Impacto ambiental do uso agrícola do lodo de esgoto. Jaguariúna: EMBRAPA Meio Ambiente, 2000 p. 259268.

5. BERTON, R.S.; CAMARGO, O. A.; VALADARES, J. M. A. S. Absorção de nutrientes pelo milho em resposta a adição de resíduos orgânicos a cinco solos paulistas. Revista Brasileira de Ciência do Solo, v.13, p.187-192, 1989.

6. BISCAIA, R. C. M.; MIRANDA, G. M. Uso do lodo de esgoto calado na produção do milho. Revista Sanare, v.5, p.8689, 1996.

7. BRASIL, Análise de corretivos, fertilizantes e inoculantes: métodos oficiais. Brasília: Ministério da Agricultura, 1988. $104 \mathrm{p}$.

8. CASTRO C.de, OLIVEIRA, F.A.de Nutrição e adubação do girassol. In: Girassol no Brasil. Londrina, PR. Editora EMBRAPA - SOJA, 2005. cap. 13, p 317- 374.

9. $\quad$ CETESB Aplicação de lodo de sistema de tratamento em áreas agrícolas; critério para projeto e operação (manual técnico). São Paulo, 1999. 32p. (CETESB NORMA P 4230).

10. DIAS, B. de O. Caracterização da matéria orgânica de Latossolo sob aplicação continuada de lodo de esgoto. Lavras UFLA, 200567 p. ( dissertação de mestrado em solos e nutrição de plantas).

11. EMPRESA BRASILEIRA DE PESQUISA AGROPECUARIA. Sistema Brasileiro de Classificação de Solos. Rio de Janeiro: EMBRAPA/CNPSO, 2006. $412 \mathrm{p}$.

12. GOMES, S.B.V.; NASCIMEN TO, C.W.A do; BIONDI, C.M.; ACCIOLY, A.M. de A. Alterações químicas em argissolo tratado com lodo de esgoto. Caatinga, Mossoró, v.18, n.3, p.185-194, 2005.

13. JORGE, F. A.; CAMARGO, A. O.; VALADARES, J. M. A. S. Condições Físicas de um Latossolo Vermelho Escuro Quatro Anos Após a Aplicação de Lodo de Esgoto e Calcário. Revista Brasileira de Ciência do Solo, v.15, p.237-240, 1991.

14. LOBO, T.F.; GRASSI FILHO, H. Sewage sludge on the development and nutricion of sunflower plants. Journal Soil Sciences Plant Nutricion, Valdivia, v.9, n.3, p. 245-255, 2009.

15. LOPES, J.C. et al. Crescimento de Digitaria ciliaris em solo tratado com lodo de esgoto bruto e corrigido. Magistra, Cruz das Almas, v. 19, n.2, p. 127-134. 2007

16. MALAVOLTA, E. Manual de calagem e adubação das principais culturas. São Paulo. Editora Ceres, 1987. 496p.

17. MELO, W.J \& MARQUES, M.O. Há perspectiva de uso nobre do esgoto. Jaboticabal Noticias, 11, p.6, 1992.

18. MELO, W.J; MARQUES, M.O.; SANTIAGO, G.; CHELLI, R.A. \& LEITE, S.A.S. Efeito de doses crescentes de resíduo orgânico sobre frações de matéria orgânica e CTC de um Latossolo cultivado com cana-de-açúcar. Revista Brasileira de Ciência do Solo, v. 18, n. 3, p. 449-455, 1994.

19. MILLER, R.H. Soil microbiology aspects of recycling sweage sludge and waste effluents on land. In: Proceding of the joint conference on recycling municipal sludge and effluent in land. Champaign, Illions, p. 79-90, 1973.

20. OLIVEIRA, F. C,; MARQUES, M. O.; BELLINGIERI, P. A.; PERCINI, D. Lodo de Esgoto como Fonte de Macronutrientes para a Cultura do Sorgo. Scientia Agrícola, Piracicaba, v.52, n.2, p.360-367, 1995.

21. PEGORINI, E.S.; ANDREOLK,C.V.; SOUZA,M.L. \& FERREIRA, A. Qualidade do lodo de esgoto utilizado na reciclagem agrícola na região metropolitana de Curitiba - PR. In: SIMPÓSIO LATINO AMERICANO DE BIOSSÓLIDO, 1., São Paulo, 2003. Anais. São Paulo, jun., 2003. 11p.

22. RAIJ, B. VAN. A capacidade de troca de cátions das frações orgânicas e minerais do solo. Bragantia, Campinas, v.28 p. 85-112, 1969

23. RAIJ B. VAN; ANDRADE,J.C.; CANTARELA,H.; QUAGGIO,J.A. - Análise Química para fertilidade de solos tropicais $1^{\text {a }}$ Edição, INSTITUTO AGRONOMICO - FUNDAÇÃO IAC, 2001. 285p.

24. RAIJ, B.V.; CANTARELLA, H.; QUAGGIO, J.A. \& FURLANI, A.M.C. Recomendações de adubação e calagem para o Estado de São Paulo, 2.ed. Campinas, Instituto Agronômico\&Fundação IAC, 1996. 285p. (Boletim técnico, 100).

25. SAS. Institute. SAS user's guide: statistics, ver 8.2; Cary, NC. 2001

26. SHIROTA, R.; ROCHA,M.T. Análise de mercado potencial agrícola do lodo de esgoto biológico resultante do tratamento de esgoto urbano na região de Limeira, SP. Piracicaba: FEALQ, 1997. 85p. (Relatório apresentado à empresa Águas de Limeira S/A.)

27. SILVA, F.C.; BOARETO A. E. ; BERTON, R. S.; ZOTELLI H. B.; PEXE, C. A.; BERNARDES, E. M. Efeito do lodo de esgoto na fertilidade de um argissolo vermelho amarelo cultivado com cana de açúcar. Pesquisa Agropecuária Brasileira, Brasília, v. 36, n.5, p. 831-840, 2001.

28. SILVA, F. C. da; BOARETTO, A. E.; BERTON, R. S.; ZOTELLI, H. B.; PEIXE, C. A.; MENDONÇA, E. Cana-deaçúcar cultivada em solo adubado com lodo de esgoto: nutrientes, metais pesados e produtividade. Pesquisa Agropecuária Brasileira, v.33, n.1, p.1-8, 1998. 
LOBO, T. F. et al. Doses de lodo de esgoto...

29. SILVA J.E., RESK, D. V. S. SHARMA, R. D. Alternativa agronômica para o biossólido: a experiência de Brasília. In BETIOL, W \& CAMARGO, O. A. (eds.) Impacto ambiental do uso agrícola do lodo de esgoto. Jaguariúna: EMBRAPA Meio Ambiente, 2000, p. 143-152.

30. SIMONETE, M. A.; KIEHL, J. D.; ANDRADE, C. A.; TEIXEIRA, C. F. Efeito do lodo de esgoto em um Argissolo e no crescimento e nutrição do milho. Pesquisa Agropecuária Brasileira, v.38, n.10, p.1187-1197, 2003.

31. TRANNIN, I. C. de B. Avaliação agronômica de um biossólido industrial e de seus efeitos sobre atributos do solo. 2004. 171p. Tese (Doutorado em Solos e Nutrição de Plantas) - Universidade Federal de Lavras (UFLA), Lavras, MG.

Recebido em 14/02/2011

Aceito em 03/05/2012 